\title{
A Systematic Approach to Identifying and Developing Implementation Strategies to Support the Uptake of a Risk Tool to Aid in Clinical Management of Patients with Syncope: A Qualitative Study
}

\section{Geneviève Rouleau}

Women's College Hospital https://orcid.org/0000-0003-1093-6577

Venkatesh Thiruganasambandamoorthy

TOH: Ottawa Hospital

Kelly Wu

Women's College Hospital

\section{Bahareh Ghaedi}

Ottawa Hospital

\section{Phuong Anh NGuyen}

Ottawa Hospital

Laura Desveaux ( $\square$ laura.desveaux@thp.ca )

Women's College Hospital https://orcid.org/0000-0003-3429-1865

\section{Research}

Keywords: emergency department, implementation strategies, physician, qualitative, syncope, user-centred design

Posted Date: December 13th, 2021

DOI: https://doi.org/10.21203/rs.3.rs-1107283/v1

License: (c) (i) This work is licensed under a Creative Commons Attribution 4.0 International License. Read Full License 


\section{Abstract}

Background: The Canadian Syncope Risk Score (CSRS) is a validated risk stratification tool developed to optimize the accuracy of emergency department decisions and inform evidence-based clinical actions. While preliminary work has been undertaken to understand the barriers to CSRS use, no work to date has explored how to implement the CSRS to overcome these barriers in practice. This study aimed to identify which implementation strategies are most appropriate to address these barriers and how they should be implemented to mitigate the possibility of poor uptake.

Methods: We conducted a series of three iterative online user-centered design workshops with emergency medicine physicians from three hospital sites in Ontario, Canada. The objective of the workshops was to engage participants in identifying acceptable strategies to promote CSRS uptake and how they should be operationalized. To support this, we systematically mapped previously identified barriers to corresponding behaviour change techniques to identify the most likely strategies to effect change. The sessions were audiorecorded and dialogue relating directly to the study objective were transcribed. We performed a qualitative content data analysis according to pre-defined objectives for each workshop.

Results: Fourteen physicians participated across the three workshops. The main implementation strategies identified to overcome identified barriers were: education in the format of meetings, videos, journal clubs, and posters (uncertainty around when and how to apply the CSRS); an online calculator and integration of the CSRS into electronic medical record (uncertainty in how to apply the CSRS), local champion (lack of team buy-in); and dissemination of evidence summaries and feedback through email communications (lack of evidence about impact).

Conclusions: The ability of the CSRS to effectively improve patient safety and syncope management relies on broad buy-in and uptake across physicians. To ensure the CSRS is well-positioned for impact, a comprehensive suite of implementation strategies was identified to address known barriers. This next phase of work will provide insight into whether these strategies facilitated better alignment with barriers, higher physician engagement with the implementation strategies, and broader uptake of the CSRS, with the objective of improving the likelihood that the CSRS will positively influence patient outcomes.

\section{Contributions To The Literature}

- Building on prior work, this study provides an example of a systematic approach to co-designing implementation strategies to support the uptake of a validated clinical decision support tool in practice.

- Our study demonstrates the importance of integrating various sources of knowledge, including theory and clinical expertise, to co-design implementation strategies tailored to real-world routines and workflows.

- Our results highlight the value of engaging participants in co-designing strategies, specifically in recognizing the need to engage an audience broader than the intended users of the clinical decision support tool.

\section{Background}


Syncope, defined as a sudden transient loss of consciousness (TLOC) followed by spontaneous complete recovery (1), is a prevalent and high-cost problem in emergency departments (ED) $(2,3)(4)$. Syncope accounts for $1-3 \%$ of ED visits, up to $6 \%$ of all hospitalizations from the ED, at an estimated cost of 2.4 billion USD for syncope related hospitalization in the United States in the year 2000 (5). In Canada, it accounts for approximately 160,000 ED annual visits, with direct hospital costs of approximately \$130 million CAD. $(6,7)$ While the cause is often benign, approximately $10 \%$ of patients will have serious underlying conditions identified within 30 days $(8,9)$. Up to half of these conditions will be identified after the initial decision to discharge or admit the patient from the ED, emphasizing the need for supports to improve risk-stratification and decision-making in the ED (1, 8-11).

The Canadian Syncope Risk Score (CSRS) is a validated risk-stratification tool to optimize the accuracy of ED decisions and inform evidence-based clinical actions $(9,12)$. Despite a robust body of evidence, no work has been undertaken to understand how to implement the CSRS in practice. Many evidence-based practices fail to demonstrate the anticipated impact due to implementation challenges, underscoring the need to proactively address barriers to uptake in order to mitigate this challenge (13-15).

A qualitative study of 41 physicians across 12 Canadian ED sites identified several barriers to uptake (16). These included concerns about continuity of care, lack of resources to support patients' follow-up including access to monitoring, workflow issues, lack of confidence in the CSRS, and lack of knowledge and skills around how to interpret and apply the criteria for various patient profiles (16). Implementation science provides a systematic approach to targeting these barriers to change $(17,18)$ and over 70 evidence-based implementation strategies that can be adapted to local contexts $(14,18)$. While we have proactively identified barriers, it remains unclear which implementation strategies are best suited to address them and how they should be operationalized to support uptake of the CSRS.

Poorly designed implementation strategies (e.g., too complex, do not adequately address known barriers) are likely to lead to suboptimal uptake, poor intervention outcomes, and failure to integrate the CSRS into practice $(14,19-21)$. Therefore, the objective of this work is to identify which strategies are most appropriate and how they should be implemented to mitigate the possibility of suboptimal implementation or poor uptake (22). The feasibility of the resulting strategies will be evaluated as part of a pilot implementation study, as the next phase of this work.

\section{Methods}

We undertook a qualitative study and we conducted a series of three iterative user-centered design workshops with ED physicians from three hospital sites in eastern Ontario (Canada) between February and April 2021, in line with guidance from French et al. (23). We used the Consolidated Criteria for Reporting Qualitative Studies (COREQ) (24) for reporting the qualitative process (Additional file 1). There is growing recognition of the complementary role and the strengths of applying user-centred design in implementation science (19-21, 2531). Both fields (user-centered design and implementation science) value and promote stakeholder engagement, specifically taking their perspectives into consideration to optimize the implementation process to effect change, and thus positive impact on population health. User-centred design engages end-users (i.e., the target audience for the CSRS) under a collaborative, participatory, and co-creative lens. It pursues the parallel goals of maximizing usability in context of those targeted by the implementation endeavour and tailoring strategies to 
users' local contexts, while retaining the core components responsible for their effectiveness (20). To prepare for the workshops, we systematically mapped previously identified barriers to corresponding behaviour change techniques (BCTs), which are "observable, replicable, and irreducible components of an intervention" to identify the most likely strategies to effect change (see Additional file 2). We prioritized the list of BCTs based on feasibility to create a short-list for discussion within the workshops. The development process of implementation strategies over the three workshops is presented in Additional file 3.

\section{Recruitment strategies}

Participant recruitment was facilitated by the main developer of the CSRS (VT) through e-mail communications with key informants at the three sites. Individuals that expressed interest in participating contacted the project lead (GR) and were provided with a study information sheet via e-mail. In each round, we aimed to recruit between five to seven ED physicians who had different knowledge levels regarding CSRS (i.e., have heard about it, had basic or good knowledge). We used a combination of convenience and purposive sampling to ensure a diversity in age, sex, years since graduation, hospital site (i.e., urban academic and non-academic), and the primary language for clinical care (English or French) in an effort to capture various perspectives.

Data collection

Zoom, a screen-share technology (32), was utilized to provide an overview of the CSRS and underlying evidence, facilitate discussion around barriers of using CSRS, and engage participants in generating strategies to overcome them. The facilitator (GR) encouraged a "think-aloud" approach (33) to provide insight into participants' thought process and gather feedback around which implementation strategies might be useful and why (or why not). Specifically, participants were asked to share their reactions, sentiments, and thought processes in real-time. Excerpt of questions used as part of the workshop facilitation guide are presented in Additional file 3. Participants filled out a brief online questionnaire via LimeSurvey (34) to capture demographic characteristics, including their job title, gender, hospital site, years of experience in career and in hospital site, and knowledge level regarding CSRS. A research assistant (KW) took notes in real time and sessions were audio-recorded to facilitate clarifying themes and insights as needed. Verbal consent was obtained at the beginning of each workshop and participants were provided with a \$100 honorarium.

\section{Data analysis}

The project lead (GR) listened to the complete audio recordings of the workshop and partially transcribed key parts of the conversation based on their relevancy to meet the workshops' objectives, i.e., quotes that shed light on the barriers and facilitators of using CSRS, as well as the strategies that would be helpful to improve its use. The project lead and the research assistant present during the workshops co-produced a preliminary summary of the findings. We performed a qualitative content data analysis (23) according to pre-defined objectives for each workshop by using a deductive and inductive process. We utilized deductive framework coding with broad categories by applying barriers previously identified barriers as deductive codes as well as theory-based strategies. We inductively coded new strategies and operationalization parameters that were suggested by the participants throughout the conversation. The research team then met to review the themes and discuss content changes. A summary of findings and proposed changes was emailed to the participants after each workshop to 
solicit feedback, validate the emerging insights and operationalization parameters, and seek clarification, if needed (member-checking) $(35,36)$.

\section{Results}

Participant characteristics

A total of 14 ED physicians participated across the three workshops and all were aware of the existence of the CSRS tool prior to participation. Participant characteristics are summarized in Table 1.

Table 1

Participant demographics

\begin{tabular}{|llll|}
\hline Characteristics & $\begin{array}{l}\text { Workshop \#1 } \\
(\mathbf{n}=5)\end{array}$ & Workshop \#2 (n=4) & $\begin{array}{l}\text { Workshop \#3 } \\
(\mathbf{n}=5)\end{array}$ \\
\hline $\begin{array}{l}\text { Gender } \\
\text { Female:Male (n) }\end{array}$ & $2: 3$ & $1: 3$ & $2: 3$ \\
\hline Years practicing medicine & $8.4(4-9)$ & $11.9(4-19.5)$ & $7(3.5-10)$ \\
Mean (range) & & & \\
\hline Site (ED) & 2 & 2 & 1 \\
\hline English academic hospital & 2 & 1 & 1 \\
\hline French academic hospital & 1 & 1 & 3 \\
\hline English non-academic hospital & & & \\
\hline
\end{tabular}

Workshop 1

Participant feedback

Participants validated the barriers identified in previous qualitative work (discomfort using the CSRS, lack of confidence, lack of knowledge and skills, and uncertainty around interpretation) and highlighted three additional barriers. These included struggling with how to apply the CSRS recommendations, inapplicability of CSRS for some patient clinical presentations, and a lack of buy-in from the broader medical team (i.e., cardiologists and internists). Physicians described that system-level barriers may prevent the ability to apply recommendations, including lack of access to outpatient cardiac monitors, difficulty keeping patients for six hours of ED observation, and the 'black hole' following patients' discharge due to unknown continuity of care.

Having a system so that when using the score, you know what's happening to the patients and what you are recommending is going to follow-through would help. The mild-risk patients I think everyone is fine with, a lot of high-risk patients we'll end up admitting, but then the high-risk that you sent home or the intermediate-risks that go home, we send to 4-5 different cardiology services and I might get a letter back from 1 out of the 10 patients that I sent. You feel like they're being sent into this black hole where you don't really necessarily have a good sense of what happens to them even though that's what you recommend. The thrombosis clinic is so helpful as 
when you don't have a CT scan at night, they get seen the next day and you can use rule to have a definitive management. But I know that it may be far beyond the scope of the rule. (Workshop\#1, Participant 5, Women)

The lack of collective buy-in is an important barrier to CSRS use, as ED physicians described the importance of all team members being aware of and having confidence in the resulting course of treatment for a given patient.

For emerg doctors you are worried to accept the responsibility of discharging people at home. I think there needs to be something coming from cardiologists to say "yes, that is acceptable to discharge someone home"; you need to know there is a timely follow-up; it is hard to adopt rule if we don't feel it is accepted widely by specialists as well. So, we feel supported to safely use it. (W\#1, P1, W)

Co-designing strategies

Participants described that effectively addressing the above barriers requires multiple strategies deployed using various dissemination channels. The need to target a broad audience (i.e., ED physicians, cardiologists, internists) with consistent exposure over time (i.e., repeat messaging) was emphasized.

Grand rounds, presentations during physicians' meetings, posters, study sheets all over the place, research assistants remind you to use it; seeing publications, seeing it on social media, pretty embedded in our group; importance of multiple strategies. (W\#1, P4, M)

Participants also highlighted the need to leverage existing structures, including integration of the CSRS into the electronic medical record (EMR), discussing the CSRS at standing educational meetings, and displaying a poster in the workplace environment. Participants felt that holding combined grand rounds with ED physicians and specialists (i.e., cardiologists and internists) would be a coordinated strategy to addressing multiple barriers simultaneously. How these educational events are promoted is important to stimulate interest and excitement, including highlighting the credibility of the speaker. Having a journal club with ED physicians and specialists to review evidence around CSRS would be useful. Podcasts can be another interesting channel to disseminate knowledge around CSRS. Participants shared the example of emergency medicine reviews and perspectives (EM: RAP), a perceived trusted online resource, which is a monthly emergency medicine audio series encompassing continuing medical education. Displaying a poster in the ED was suggested as a helpful visual cue, with participants describing the usefulness of the CT head rule poster $(37,38)$ as an example.

Flow diagram of what you would do with each category; I think a lot of people in emergency medicine like "if this, then that," to know which way to flow. That can help to take some of the thought process out if it as long as it is standardized across colleagues/specialists. We want to be practicing along with our colleagues and specialists, so having consensus with colleagues to follow the diagram with appropriate clinical practice and applying the rule appropriately, I think would help too. (W\#1, P4, M)

Resulting action

The research team created two prototype posters for discussion in workshop 2 (Additional file 5). The two posters encompassed similar content with different displays. In poster \#1, information around "For whom the CSRS must be applied" and "When to use CSRS" was highlighted. Poster \#2 focused on the proposed course of treatment prior to the application of the CSRS. In both prototype posters, the CSRS was illustrated along with 
the three risk levels and their proposed practice-based recommendations. In response to the need for education about the CSRS, we distributed eight previously developed educational videos to participants of workshop 2 (Additional file 6). We asked participants to focus on the four videos that provided 1) an overview of CSRS, as well as the risk of serious adverse events and the recommendations for 2) low, 3) medium, and 4) high-risk patients.

Workshop 2

Participant feedback on barriers

Participants described the inapplicability of CSRS for some clinical presentations, a lack of buy-in from the broader medical team, and discomfort using CSRS. One participant explained the source of discomfort in using the tool as a feeling of hesitancy:

We reviewed this in journal club at [our hospital] and the biggest thing that came up and common to clinical decision rule is the clinical Gestalt at the end (...) Physicians felt hesitant using tool arguing it is telling me if it is vasovagal or cardiac syncope, seems counterintuitive to make decision in order to use tool to tell you something you already know. (W\#2, P2, W)

Participants also highlighted the lack of evidence about how the CSRS practice-based recommendations impact patients' outcomes as a barrier to uptake.

Co-designing strategies

Educational meetings (grand rounds) and materials (educational videos) were discussed extensively during the second workshop as relevant implementation strategies. Participants emphasized the need to expand the target of the implementation efforts and to onboard the head of department and nursing staff, in addition to physicians. There was agreement that educational meetings could be utilized to promote general awareness of the CSRS and to encourage a nuanced discussion about its application. However, a range of opinions were expressed on the best format for those educational meetings (e.g., combined grand rounds, case-based discussion in small group). Participants also highlighted the need for a local champion to model the application and use of the CSRS and further influence uptake among colleagues.

I think case-based rounds is great. I think to get hospital buy-in I'm thinking smaller community hospitals. I think having combined rounds with cardiology, medicine, and emerg to go over the score and how to apply it, and what are monitoring implications - I think that's helpful as a group. That way the discussion happens with all the key players and the barriers to implementing this. As opposed to presenting it in silos really when a patient comes all these people are important, so combining a strategy could be helpful. (W\#2, P2, W)

Excerpt of the educational videos were played during the workshop to solicit individual reactions and group discussion. All the components and features (e.g., written summary, questions, graphics, videos, and links to scientific papers) were perceived as useful and the content was perceived as clear, concise, relevant, and credible. The duration of videos was reasonable if viewed out of the workplace but were too long to be viewed during a shift. It was suggested that a five-minute video that includes the main information would be an ideal length and would facilitate wider dissemination of the CSRS. 
When reviewing the two poster prototypes, participants suggested the need to simplify the posters, separating the explicative notes (i.e., additional information) from the care pathway and move those notes as footnotes using a different font (e.g., smaller fonts for footnotes) to make the content easier to read, and adding QR codes to facilitate immediate access to the CSRS online calculator.

Resulting action

We prepared a brief written summary of evidence (three short paragraphs) to support each practice recommendation for each patient group (low, medium and high-risk) (Additional file 7) and re-designed the posters in response to feedback and added three QR codes - 1) How to use CSRS, 2) Recommendation evidence, and 3) Online calculator. We also pulled e-mail communication (Additional file 9) from a previous pilot study [Remote Cardiac Monitoring of Higher-Risk Emergency Department Syncope Patients after Discharge (REMOSYNC) study; yet to be published], as an example to communicate positive feedback on patient impact (i.e., an example where home monitoring detected a patient arrythmia) as well as messaging to remind physicians to use the CSRS.

Workshop 3

Co-designing strategies

Participants discussed the perceived usability, usefulness, and operationalization of the following strategies: the online calculator, the summary of evidence, the poster, the local champion, and the e-mail communication.

All participants tested the CSRS online calculator prior to the workshop (Additional file 8). Participants suggested ways of improving the usability of the online calculator: 1) reviewing the wording of some criteria to avoid misleading interpretations, 2) adding a "not drawn" response option to this question "elevated troponin level", 3) adding an access to evidence, and 4) adding access to practice-based recommendations for low, medium, and high-risk patients (i.e., what to do with the risk score). All participants had an intention to use the online calculator but for different purposes: utilization in practice and as an education tool with medical students. Some would use it only if it is integrated into EMR and will not use it if it is part of a mobile application.

Participants would find it useful to discuss in length the summary of evidence in grand rounds or in another type of educational meeting, as an initial evidence uptake. Getting easy access to evidence was considered important: tying evidence to online calculator and to EMR would be one way to improve its access.

Thinking back to other scores, or decision rules that are on calculator... It does bug me sometimes when I'm not able to access like a summary of why that's the recommendation or why that's the rule but again having an optional because if you already know it you don't need to come up every time if you forget or you want to know about the medium risk what exactly are the details having the option to go easily access from the rule would be nice. $(\mathrm{W} \# 3, \mathrm{P} 5, \mathrm{~W})$

Participants shared their reactions towards the second prototype poster and found it usable (i.e., easy to follow, simple, and appealing (nice colors)). They would use it as a reminder and as a prompt to apply CSRS. They 
would also refer their colleagues to this poster, which is seen as a way of giving credibility for their ED syncope management course of treatment.

I can refer to that poster, maybe give me a little bit of credibility if I'm advocating for an admission where I'm getting pushed back. (W\#3, P2, M)

Participants highlighted that such a type of poster would be helpful, especially at the early stages of the CSRS implementation process in ED. However, participants identified some barriers of using such a poster: risk of poster fatigue and no space to display it in their clinical settings. They would probably not use the QR codes for these reasons: they assumed that if they used CSRS, they would already be familiar with the underlying evidence (so no need to scan QR codes for additional information); and perceived lack of skills of using QR codes. If one QR code had to be kept within the poster, it would be the online calculator.

Participants perceived that the local champion could play multiple roles: speaker in educational meetings, connection between the clinical and research team, monitors the implementation process over time, and provides in-person and/or written feedback:

After the six months check-up can be within the department if you have that champion, is the one that can do that link up. The first six months I think will give you enough information, does that local champion can be the one and that links back with the research team and see what is it at that point, having someone locally I think is significantly better to get like off the cuff comments and things like that and how they wish it was changed, applied or supports, I think it has better chance of getting quality feedback and regular feedback. (W\#3, P3, M)

Finally, e-mail communication with feedback would be useful for ED physicians to convince them to use CSRS. Participants had different opinions on how and by whom feedback could be delivered, such as through educational outreach, one-to-one discussion with local champion, and e-mail communication. They would like to be provided feedback by the research team (especially the CSRS developer) and by a cardiologist within their hospital. This quote speaks to quality indicators that would be of interest:

I think for me anecdotal feedback is really helpful. So with the implementation of like the electronic records and EPIC (EMR), actually getting responses from the referrals that I make and similarly like for this type of thing, getting even anecdotal or like $\mathrm{n}$ of 5 feedback from cardiology on the results of the Holter monitor well, over time, I think, build up to convince me to use the rule. So I think that there should be someone at each site who's trying to collect that information, like, based on what was the risk level patient has, did they have a Holter or not? And are the numbers that we're seeing, matching up what the what the actual CSRS showed. (W\#3, P5, W)

Resulting action

We synthesized the findings across the three workshops and selected the strategies to be developed and deployed as part of a future pilot study. The parameters of these strategies are outlined in Table 2. 
Table 2

Summary of the strategies and their parameters over the three workshops

\begin{tabular}{|c|c|c|c|c|c|}
\hline Strategy & Required content & $\begin{array}{l}\text { Mode of } \\
\text { delivery }\end{array}$ & $\begin{array}{l}\text { Delivery } \\
\text { source }\end{array}$ & $\begin{array}{l}\text { Target } \\
\text { audience }\end{array}$ & $\begin{array}{l}\text { Target } \\
\text { outcome }\end{array}$ \\
\hline $\begin{array}{l}\text { Educational } \\
\text { meetings (e.g., } \\
\text { grand rounds, } \\
\text { case-based } \\
\text { rounds, case } \\
\text { discussion) }\end{array}$ & $\begin{array}{l}\text { Nuances, barriers, } \\
\text { and pitfalls when } \\
\text { using CSRS; } \\
\text { evidence } \\
\text { underlying CSRS } \\
\text { and } \\
\text { recommendations; } \\
\text { cost/resources } \\
\text { (e.g., cardiac } \\
\text { monitors) }\end{array}$ & $\begin{array}{l}\text { Online } \\
\text { In-person }\end{array}$ & $\begin{array}{l}\text { CSRS } \\
\text { experts, } \\
\text { cardiology, } \\
\text { general } \\
\text { medicine }\end{array}$ & $\begin{array}{l}\text { All locations } \\
\text { where CSRS } \\
\text { will be } \\
\text { applied } \\
\text { Diverse } \\
\text { stakeholders }^{\text {a }}\end{array}$ & $\begin{array}{l}\text { Improve } \\
\text { knowledge of } \\
\text { and comfort } \\
\text { in using CSRS } \\
\text { Improve skills } \\
\text { on how to use } \\
\text { CSRS }\end{array}$ \\
\hline $\begin{array}{l}\text { Educational } \\
\text { videos }\end{array}$ & $\begin{array}{l}\text { How to deal with } \\
\text { ultra-low-risk } \\
\text { criteria and } \\
\text { troponin; what to } \\
\text { do with risk score }\end{array}$ & Online & $\begin{array}{l}\text { CSRS } \\
\text { experts }\end{array}$ & & \\
\hline Journal clubs & $\begin{array}{l}\text { Scientific papers - } \\
\text { development and } \\
\text { validation of CSRS }\end{array}$ & Online & $\begin{array}{l}\text { CSRS } \\
\text { experts }\end{array}$ & $\begin{array}{l}\text { Medical } \\
\text { staff }^{b}\end{array}$ & $\begin{array}{l}\text { Improve } \\
\text { knowledge } \\
\text { about } \\
\text { evidence }\end{array}$ \\
\hline $\begin{array}{l}\text { Online } \\
\text { calculator }\end{array}$ & $\begin{array}{l}\text { How to deal with } \\
\text { troponin criterion; } \\
\text { what to do with the } \\
\text { risk score } \\
\text { (recommendations) }\end{array}$ & $\begin{array}{l}\text { Webc, mobile } \\
\text { application, } \\
\text { EMR }\end{array}$ & $\begin{array}{l}\text { Not } \\
\text { applicable }\end{array}$ & CSRS users $^{d}$ & $\begin{array}{l}\text { Improve CSRS } \\
\text { integration } \\
\text { into workflow }\end{array}$ \\
\hline $\begin{array}{l}\text { CSRS } \\
\text { integration } \\
\text { into EMR }\end{array}$ & $\begin{array}{l}\text { Interpretation of the } \\
\text { risk score; what to } \\
\text { do with the risk } \\
\text { score }\end{array}$ & EMR & & & \\
\hline
\end{tabular}

a - Emergency medicine physicians, family physicians working at ED, any consultants who are asked for high-risk patients, cardiologists, internists, nurses (including nurse practitioner), support from head of department

b - Internal medicine, cardiology and emergency medicine physicians

c - MDCalc (53)

d - All emergency medicine physicians and residents 


\begin{tabular}{|c|c|c|c|c|c|}
\hline Strategy & Required content & $\begin{array}{l}\text { Mode of } \\
\text { delivery }\end{array}$ & $\begin{array}{l}\text { Delivery } \\
\text { source }\end{array}$ & $\begin{array}{l}\text { Target } \\
\text { audience }\end{array}$ & $\begin{array}{l}\text { Target } \\
\text { outcome }\end{array}$ \\
\hline \multirow[t]{2}{*}{$\begin{array}{l}\text { Local } \\
\text { champion }\end{array}$} & $\begin{array}{l}\text { Roles: Speaker, } \\
\text { monitor the } \\
\text { implementation } \\
\text { process, support } \\
\text { the teams; adapt } \\
\text { and tailor } \\
\text { implementation } \\
\text { strategies; provide } \\
\text { feedback. }\end{array}$ & In-person & \multirow[t]{2}{*}{$\begin{array}{l}\text { Local } \\
\text { emergency } \\
\text { medicine } \\
\text { physicians } \\
\text { and } \\
\text { cardiologists } \\
\text { (each site) }\end{array}$} & \multirow[t]{2}{*}{ CSRS users $^{d}$} & \multirow[t]{2}{*}{$\begin{array}{l}\text { Improve } \\
\text { collective buy- } \\
\text { in }\end{array}$} \\
\hline & $\begin{array}{l}\text { Preferred attributes: } \\
\text { Strong leadership } \\
\text { skills, know how to } \\
\text { apply CSRS and } \\
\text { recommendations, } \\
\text { positive influence } \\
\text { on colleagues. }\end{array}$ & & & & \\
\hline \multirow[t]{2}{*}{ Poster } & \multirow{2}{*}{$\begin{array}{l}\text { Care pathway, how } \\
\text { to deal with } \\
\text { troponin criterion, } \\
\text { recommendations }\end{array}$} & Paper & \multirow{2}{*}{$\begin{array}{l}\text { Not } \\
\text { applicable }\end{array}$} & \multirow{2}{*}{$\begin{array}{l}\text { Diverse } \\
\text { stakeholders }^{a}\end{array}$} & \multirow{2}{*}{$\begin{array}{l}\text { Improve } \\
\text { collective buy- } \\
\text { in }\end{array}$} \\
\hline & & QR codes & & & \\
\hline \multirow[t]{2}{*}{$\begin{array}{l}\text { Dissemination } \\
\text { of evidence } \\
\text { summary }\end{array}$} & \multirow{2}{*}{$\begin{array}{l}\text { Impact of CSRS } \\
\text { practice-based } \\
\text { recommendations } \\
\text { on patients' } \\
\text { outcomes }\end{array}$} & $\begin{array}{l}\text { Online } \\
\text { calculator, QR } \\
\text { codes, }\end{array}$ & \multirow[t]{2}{*}{$\begin{array}{l}\text { Electronic } \\
\text { content }\end{array}$} & \multirow[t]{2}{*}{$\begin{array}{l}\text { Diverse } \\
\text { stakeholders }^{\mathrm{a}}\end{array}$} & \multirow[t]{2}{*}{$\begin{array}{l}\text { Improve } \\
\text { knowledge }\end{array}$} \\
\hline & & In-person & & & \\
\hline Feedback & $\begin{array}{l}\text { CSRS impacts on } \\
\text { providers' practice; } \\
\text { numbers of cardiac } \\
\text { monitor referrals } \\
\text { and of arrythmias } \\
\text { detected }\end{array}$ & $\begin{array}{l}\text { In-person and } \\
\text { written }\end{array}$ & Champions & CSRS users $^{d}$ & $\begin{array}{l}\text { Improve skills } \\
\text { and adoption } \\
\text { of behaviour } \\
\text { (CSRS } \\
\text { uptake) }\end{array}$ \\
\hline \multirow[t]{2}{*}{ Prompts } & \multirow{2}{*}{$\begin{array}{l}\text { Invitation to use } \\
\text { CSRS, image with } \\
\text { arrythmia detected } \\
\text { (feedback) }\end{array}$} & \multirow[t]{2}{*}{$\begin{array}{l}\text { Email } \\
\text { communication }\end{array}$} & $\begin{array}{l}\text { CSRS } \\
\text { experts }\end{array}$ & \multirow[t]{2}{*}{ CSRS users $^{d}$} & \multirow{2}{*}{$\begin{array}{l}\text { Remind } \\
\text { physicians to } \\
\text { use CSRS and } \\
\text { its positive } \\
\text { consequences }\end{array}$} \\
\hline & & & Champions & & \\
\hline \multicolumn{6}{|c|}{$\begin{array}{l}\text { a - Emergency medicine physicians, family physicians working at ED, any consultants who are asked for } \\
\text { high-risk patients, cardiologists, internists, nurses (including nurse practitioner), support from head of } \\
\text { department }\end{array}$} \\
\hline \multicolumn{6}{|c|}{ b - Internal medicine, cardiology and emergency medicine physicians } \\
\hline \multicolumn{6}{|l|}{$c-$ MDCalc (53) } \\
\hline \multicolumn{6}{|c|}{ d - All emergency medicine physicians and residents } \\
\hline
\end{tabular}

\section{Discussion}

Main findings 
This work built on a qualitative understanding of perceived barriers to implementing the CSRS (16) to identify which evidence-based strategies should be used and how they can be operationalized to overcome these barriers. Key barriers included uncertainty around when and how to apply the CSRS recommendations, lack of resources (e.g., cardiac monitors), lack of buy-in from the broader medical team, discomfort (hesitancy) using CSRS, and lack of evidence about the impact on patient outcomes. Surprisingly, no reference on workload or time constraint was brought up, as is often found in other studies (39-42). Our findings suggest that physician capability should be a central target of implementation supports, specifically the capability to interpret CSRSbased criteria and apply it across a range of clinical presentations.

Training is an evidence-based and frequently utilized strategy to build physician capability by increasing their skills (43). This can be operationalized through a variety of mechanisms, including seminars, interactive workshops, and teaching programmes such as simulation, and training sessions $(44,45)$. In our study, participants largely referred to educational meetings (e.g., combined grand rounds inclusive of all relevant specialities) and educational videos. Skill-building can be supplemented by creating increased opportunity to utilize the CSRS, including integration into the EMR, engaging local champions, displaying posters, and sending e-mail communications to encourage use $(41,42,46)$. This suite of strategies are commonly used in the ED setting (46) and have demonstrated effectiveness in promoting guideline-adherent care (47).

Achieving collective buy-in across multiple speciality groups (i.e., ED, cardiology, and internal medicine) was highlighted as an essential condition for successful uptake of the CSRS. While the importance of this broader support is well-documented $(42,48)$, including in the area of risk stratification $(42)$, the importance of designing implementation strategies targeting this broader audience (i.e., an audience beyond the immediate end-user) has been unexplored. While ED syncope care primarily rests on the shoulders of the emergency physician, support from experts in cardiology, internal medicine, and hospitalists is needed for care of those with suspected/identified serious conditions, and further inpatient or outpatient investigations. Physicians rely on their colleagues and professional networks as a unique source of tacit knowledge that serve to either validate initial reasoning or offer alternative approaches (49). This presents an opportunity to influence uptake through existing channels of social influence that extend beyond the primary setting of interest (in this case, the ED). Strategies may benefit from alignment with the underlying factors that influence patterns of collaboration, including perceived reputational value, experiential information (including personal relationships and visibility), professional identity, and self-awareness of competence (50). In addition, strategies that target components such as champion/opinion leader, social support, and credible source would be promising ingredients to consider (51).

Similar to the Bravo et al. (52) study, our findings highlight a tension between user preferences and scientific evidence and the critical role of triangulating user input alongside scientific evidence to leverage both sources of knowledge in the design of implementation strategies. Specifically, physician participants identified education in the form of grand rounds as a strategy to address areas of uncertainty and to improve collective awareness of the tool within the interdisciplinary team. While education effectively increases knowledge and awareness, it is training that effectively builds or strengthens skills (43). Based on physicians' perceived barrier of not knowing how to apply CSRS, education alone might not be sufficient because it has to do with developing abilities to apply the tool among various patients. In that case, training would be more suited. 
Simply put: each strategy has its own function and mechanisms allowing it to overcome barriers of using CSRS and strengthen the facilitators.

\section{Limitations}

We used a combination of purposive and convenience sampling to recruit ED physicians working at three different hospitals, therefore the results may not reflect the experiences of physicians working in other sites. Further, all participants were aware of the development of the CSRS prior to their participation in the workshops, with most being employed at the same hospital where the CSRS has been piloted. Future work should explore whether the resulting strategies align with and effectively address the barriers experienced by those who are unaware of the CSRS. Finally, while a comprehensive list of potential behaviour change techniques was developed (Additional file 2), only a subset of these were prioritized for discussion in the workshops due to feasibility and time constraints. A more comprehensive discussion would have yielded additional strategies to address the identified barriers and future work should assess whether barriers persist that might be amenable to strategies that were not thoroughly considered. For example, we could operationalize more extensively the identification and the preparation of local champions, and we could target which skills would need to be addressed in a training and how we should impart them (e.g., simulation, small-group workshop with demonstration on how using CSRS with different patients' clinical presentation). The provision of performance feedback on the accurate use of the CSRS for risk-stratifying patients by expert (e.g., cardiologist, CSRS developer) could also be an avenue to consider.

\section{Conclusion}

The ability of the CSRS to effectively improve patient safety and ED syncope management relies on broad buyin and uptake across ED physicians. To ensure the CSRS is well-positioned for impact, a comprehensive suite of implementation strategies was identified to address known barriers. We have prioritized the following implementation strategies to be pilot tested in two clinical settings: posters, educational meetings (grand rounds), educational videos (with a training component on how to apply CSRS among various patients), integration of the CSRS into EMR and access to an online calculator to calculate risk score. These strategies will be evaluated to understand whether and how they are being implemented in practice, if they are effective in addressing the identified barriers, and if they are targeting the hypothesized outcome. This next phase of work will provide insight into whether this user-centred design approach facilitated better alignment with preidentified barriers, higher physician engagement with the implementation strategies, and broader uptake of the CSRS, with the objective of improving the likelihood that the CSRS will positively influence the outcomes among ED patients with syncope.

\section{Abbreviations}

\section{BCT}

Behavioural change techniques

COM-B

Capability, Opportunity, Motivation - Behaviour

CSRS

Canadian Syncope Risk Score

Page 13/19 
CT

computed tomography

ED

emergency department

EMR

electronic medical record

EM

RAP:emergency medicine reviews and perspectives

IF

Intervention function

MD

Medical doctor

QR codes

Quick response codes

TLOC

transient loss of consciousness

REMOSYNC

Remote Cardiac Monitoring of Higher-Risk Emergency Department Syncope Patients after Discharge study TDF

theoretical domain framework

\section{Declarations}

\section{Ethics approval and consent to participate}

This study received approval from the Women's College Hospital Assessment Process for Quality Improvement Projects (APQIP - REB \# 2020-0143-E) pathway for institutional review (the project was deemed to be exempt from REB review). All participants provided informed consent.

\section{Consent for publication}

Not applicable

\section{Competing interests}

The authors declare that they have no competing interests.

\section{Availability of data and materials}

The datasets used and/or analysed during the current study are available from the corresponding author on reasonable request.

\section{Funding}

This study received funding from the Cardiac Arrhythmia Network of Canada (CANet). The opinions, results, and conclusions reported in this paper are those of the authors and are independent from the funding sources. 
During the study, Dr. Desveaux's research was supported by the Canadian Institutes of Health Research. Dr. Thiruganasambandamoorthy holds a salary award from the Physicians' Services Incorporated (PSI) Foundation's 'PSI-50 Mid-Career Clinical Research Award' and a Tier-1 Clinical Research Chair in Cardiovascular Emergencies through the Faculty of Medicine, University of Ottawa.

\section{Authors' contributions}

GR and LD conceived and designed the study. VT validated and approved the study design. GR collected the data, analyzed the data and interpreted them under LD's supervision. GR and LD drafted the paper. All authors (GR, VT, KW, BG, PAN, LD) revised the manuscript, approved the submitted version have agreed both to be personally accountable for the author's own contributions and to ensure that questions related to the accuracy or integrity of any part of the study, even ones in which the author was not personally involved, are appropriately investigated, resolved, and the resolution documented in the literature.

\section{Acknowledgements}

We are deeply indebted to all emergency physicians at the study sites who participated in that work. We acknowledge the following people: Dr Maria Keller, Dr. Christopher Fabian, Dr. Bikalpa Khatiwada, and Natasha Hudek. We are grateful to Marlena Dang Nguyen for her inputs in the early stages of the project.

\section{References}

1. Brignole M, Moya A, de Lange FJ, Deharo JC, Elliott PM, Fanciulli A, et al. 2018 ESC Guidelines for the diagnosis and management of syncope. Eur Heart J. 2018;19:19.

2. Brignole M, Menozzi C, Bartoletti A, Giada F, Lagi A, Ungar A, et al. A new management of syncope: prospective systematic guideline-based evaluation of patients referred urgently to general hospitals. Eur Heart J. 2006;27(1):76-82.

3. Sandhu RK, Sheldon RS. Syncope in the Emergency Department. Front Cardiovasc Med. 2019;6:180.

4. Soteriades ES, Evans JC, Larson MG, Chen MH, Chen L, Benjamin EJ, et al. Incidence and prognosis of syncope. N Engl J Med. 2002;347(12):878-85.

5. Sun BC, Emond JA, Camargo CA, Jr. Direct medical costs of syncope-related hospitalizations in the United States. Am J Cardiol. 2005;95(5):668-71.

6. Thiruganasambandamoorthy V, Hess EP, Turko E, Perry JJ, Wells GA, Stiell IG. Outcomes in Canadian emergency department syncope patients-are we doing a good job? J Emerg Med. 2013;44(2):321-8.

7. Sandhu RK, Tran DT, Sheldon RS, Kaul P. A Population-Based Cohort Study Evaluating Outcomes and Costs for Syncope Presentations to the Emergency Department. JACC Clin Electrophysiol. 2018;4(2):265-73.

8. Solbiati M, Bozzano V, Barbic F, Casazza G, Dipaola F, Quinn JV, et al. Outcomes in syncope research: a systematic review and critical appraisal. Intern Emerg Med. 2018;13(4):593-601.

9. Thiruganasambandamoorthy V, Kwong K, Wells GA, Sivilotti ML, Mukarram M, Rowe BH, et al. Development of the Canadian Syncope Risk Score to predict serious adverse events after emergency department assessment of syncope. CMAJ. 2016;188(12):E289-98. 
10. Graham I, Stiell I, McAuley L, et al. Potential areas for new clinical decision rules: comparison of North America and Europe [Abstract]. Acad Emerg Med. 1999;15(6):433.

11. Shen WK, Sheldon RS, Benditt DG, Cohen MI, Forman DE, Goldberger ZD, et al. 2017 ACC/AHA/HRS Guideline for the Evaluation and Management of Patients With Syncope: Executive Summary: A Report of the American College of Cardiology/American Heart Association Task Force on Clinical Practice Guidelines and the Heart Rhythm Society. J Am Coll Cardiol. 2017;70(5):620-63.

12. Thiruganasambandamoorthy V, Sivilotti MLA, Le Sage N, Yan JW, Huang P, Hegdekar M, et al. Multicenter Emergency Department Validation of the Canadian Syncope Risk Score. J Am Med Assoc Int Med. 2020;180(5):737-44.

13. Curran GM. Implementation science made too simple: a teaching tool. Implementation Sci Comm [Internet]. 2020; 1(1).

14. Damschroder LJ, Aron DC, Keith RE, Kirsh SR, Alexander JA, Lowery JC. Fostering implementation of health services research findings into practice: a consolidated framework for advancing implementation science. Implementation Sci. 2009;4:50.

15. Mallonee S, Fowler C, Istre GR. Bridging the gap between research and practice: a continuing challenge. Injury prevention: journal of the International Society for Child and Adolescent Injury Prevention. 2006;12(6):357-9.

16. Hudek N, Brehaut J, Thiruganasambandamoorthy V. Identification of barriers and facilitators for implementation of the Canadian Syncope Risk Score. Unpublished work.

17. Effective Practice and Organisation of Care (EPOC). EPOC Taxonomy 2015 [Available from: epoc.cochrane.org/epoc-taxonomy.

18. Cahill LS, Carey LM, Lannin NA, Turville M, O'Connor D. Implementation interventions to promote the uptake of evidence-based practices in stroke rehabilitation. Cochrane Database Syst Rev. 2017(3).

19. Dopp AR, Parisi KE, Munson SA, Lyon AR. A glossary of user-centered design strategies for implementation experts. Transl Behav Med. 2019;9(6):1057-64.

20. Dopp AR, Parisi KE, Munson SA, Lyon AR. Integrating implementation and user-centred design strategies to enhance the impact of health services: protocol from a concept mapping study. Health Res Policy Syst. 2019;17(1):1.

21. Lyon AR, Koerner K. User-Centered Design for Psychosocial Intervention Development and Implementation. Clin Psychol (New York). 2016;23(2):180-200.

22. Proctor EK, Silmere H, Raghavan R, Hovmand P, Aarons G, Bunger A, et al. Outcomes for implementation research: conceptual distinctions, measurement challenges, and research agenda. Adm Policy Ment Health. 2011;38(2):65-76.

23. French SD, Green SE, O'Connor DA, McKenzie JE, Francis JJ, Michie S, et al. Developing theory-informed behaviour change interventions to implement evidence into practice: a systematic approach using the Theoretical Domains Framework. Implementation Sci. 2012;7(1):38.

24. Tong A, Sainsbury P, Craig J. Consolidated criteria for reporting qualitative research (COREQ): a 32-item checklist for interviews and focus groups. Int J Qual Health Care. 2007;19(6):349-57.

25. Bunce AE, Gruß I, Davis JV, Cowburn S, Cohen D, Oakley J, et al. Lessons learned about the effective operationalization of champions as an implementation strategy: results from a qualitative process 
evaluation of a pragmatic trial. Implementation Sci. 2020;15(1):87.

26. Chen E, Neta G, Roberts MC. Complementary approaches to problem solving in healthcare and public health: implementation science and human-centered design. Transl Behav Med. 2020.

27. Danitz SB, Stirman SW, Grillo AR, Dichter ME, Driscoll M, Gerber MR, et al. When user-centered design meets implementation science: integrating provider perspectives in the development of an intimate partner violence intervention for women treated in the United States' largest integrated healthcare system. BMC Womens Health. 2019;19(1):145.

28. Dopp AR, Parisi KE, Munson SA, Lyon AR. Aligning implementation and user-centered design strategies to enhance the impact of health services: results from a concept mapping study. Implementation Sci Comm. 2020;1(1):17.

29. Gold R, Bunce A, Cowburn S, Davis JV, Nelson JC, Nelson CA, et al. Does increased implementation support improve community clinics' guideline-concordant care? Results of a mixed methods, pragmatic comparative effectiveness trial. Implementation Sci. 2019;14(1):100.

30. Landis-Lewis Z, Kononowech J, Scott WJ, Hogikyan RV, Carpenter JG, Periyakoil VS, et al. Designing clinical practice feedback reports: three steps illustrated in Veterans Health Affairs long-term care facilities and programs. Implementation Sci. 2020;15(1):7.

31. Witteman HO, Dansokho SC, Colquhoun H, Coulter A, Dugas M, Fagerlin A, et al. User-centered design and the development of patient decision aids: protocol for a systematic review. Syst Rev. 2015;4(1).

32. Zoom Video Communications Inc. Security guide 2021 [Available from: https://explore.zoom.us/docs/doc/Zoom-Security-White-Paper.pdf.

33. Fonteyn ME, Kuipers B, Grobe SJ. A Description of Think Aloud Method and Protocol Analysis. Qual Health Res. 1993;3(4):430-41.

34. LimeSurvey Development Team. LimeSurvey - The free and open source survey software tool. 2021.

35. Cypress BS. Rigor or Reliability and Validity in Qualitative Research: Perspectives, Strategies, Reconceptualization, and Recommendations. Dimens Crit Care Nurs. 2017;36(4):253-63.

36. Lincoln YS, Guba EG. Naturalistic inquiry. Thousand Oaks, CA: Sage Publications; 1985.

37. Lewis D. New SJRHEM Guideline - Minor Head Injury 2014 [Available from: http://sjrhem.ca/new-sjrhemguideline-minor-head-injury/.

38. Stiell IG, Wells GA, Vandemheen K, Clement C, Lesiuk H, Laupacis A, et al. The Canadian CT Head Rule for patients with minor head injury. Lancet. 2001;357(9266):1391-6.

39. Kingston M, Griffiths R, Hutchings H, Porter A, Russell I, Snooks H. Emergency admission risk stratification tools in UK primary care: a cross-sectional survey of availability and use. Br J Gen Pract. 2020;70(699):e740-e8.

40. Muthee TB, Kimathi D, Richards GC, Etyang A, Nunan D, Williams V, et al. Factors influencing the implementation of cardiovascular risk scoring in primary care: a mixed-method systematic review. Implementation Sci. 2020;15(1):57.

41. Wilson M, Keeley J, Kingman M, Wang J, Rogers F. Current clinical utilization of risk assessment tools in pulmonary arterial hypertension: a descriptive survey of facilitation strategies, patterns, and barriers to use in the United States. Pulm Circ. 2020;10(3):2045894020950186. 
42. Westafer LM, Kunz A, Bugajska P, Hughes A, Mazor KM, Schoenfeld EM, et al. Provider Perspectives on the Use of Evidence-based Risk Stratification Tools in the Evaluation of Pulmonary Embolism: A Qualitative Study. Acad Emerg Med. 2020;27(6):447-56.

43. Michie S, van Stralen MM, West R. The behaviour change wheel: A new method for characterising and designing behaviour change interventions. Implementation Sci. 2011;6(1):42.

44. Gamage USH, Mahesh PKB, Schnall J, Mikkelsen L, Hart JD, Chowdhury H, et al. Effectiveness of training interventions to improve quality of medical certification of cause of death: systematic review and metaanalysis. BMC Med. 2020;18(1):384.

45. Lateef F. Simulation-based learning: Just like the real thing. J Emerg Trauma Shock. 2010;3(4):348-52.

46. de Wit K, Curran J, Thoma B, Dowling S, Lang E, Kuljic N, et al. Review of implementation strategies to change healthcare provider behaviour in the emergency department. Can J Em Med. 2018;20(3):453-60.

47. Ebben RHA, Siqeca F, Madsen UR, Vloet LCM, van Achterberg T. Effectiveness of implementation strategies for the improvement of guideline and protocol adherence in emergency care: a systematic review. BMJ Open. 2018;8(11):e017572.

48. Dmitriew C, Ohle R. Barriers and facilitators affecting implementation of the Canadian clinical practice guidelines for the diagnosis of acute aortic syndrome. Implementation Sci Comm. 2021;2(1):60.

49. Cohen DA, Levy M, Cohen Castel O, Karkabi K. The influence of a professional physician network on clinical decision making. Patient Educ Couns. 2013;93(3):496-503.

50. Kierkegaard P, Owen-Smith J. Determinants of physician networks: an ethnographic study examining the processes that inform patterns of collaboration and referral decision-making among physicians. BMJ Open. 2021;11(1):e042334.

51. Michie S, Richardson M, Johnston M, Abraham C, Francis J, Hardeman W, et al. The behavior change technique taxonomy (v1) of 93 hierarchically clustered techniques: building an international consensus for the reporting of behavior change interventions. Ann Behav Med. 2013;46(1):81-95.

52. Bravo CA, Llovet D, Witteman HO, Desveaux L, Presseau J, Saragosa M, et al. Designing Emails Aimed at Increasing Family Physicians' Use of a Web-Based Audit and Feedback Tool to Improve Cancer Screening Rates: Cocreation Process. JMIR Human Factors. 2018;5(3):e25.

53. Thiruganasambandamoorthy V. MDCalc: Canadian Syncope Risk Score 2021 [Available from: https://www.mdcalc.com/canadian-syncope-risk-score.

54. Carey RN, Connell LE, Johnston M, Rothman AJ, de Bruin M, Kelly MP, et al. Behavior Change Techniques and Their Mechanisms of Action: A Synthesis of Links Described in Published Intervention Literature. Ann Behav Med. 2019;53(8):693-707.

55. Crane D. Behaviour Change Technique Taxonomy v1 (BCTTv1) app 2013 [Available from: https://apps.apple.com/gb/app/bct-taxonomy/id871193535.

56. Michie S, Johnston M, Francis J, Hardeman W, Eccles M. From Theory to Intervention: Mapping Theoretically Derived Behavioural Determinants to Behaviour Change Techniques. Appl Psychol. 2008;57(4):660-80.

57. Human Behaviour-Change Project. The Theory and Techniques Tool [Available from: https://theoryandtechniquetool.humanbehaviourchange.org/. 
58. D'Lima D, Lorencatto F, Michie S. Handbook on Implementation Science. The Behaviour Change Wheel approach: Edward Elgar Publishing; 2020.

\section{Supplementary Files}

This is a list of supplementary files associated with this preprint. Click to download.

- Additionalfile1.COREQ.docx

- Additionalfile2.BCT.docx

- Additionalfile3.Developmentprocess.docx

- Additionalfile4.Facilitationguide.docx

- Additionalfile5Prototypeposters.docx

- Additionalfile6.Educationalvideos.docx

- Additionalfile7.Writtensummaryofevidence.docx

- Additionalfile8.Onlinecalculator.docx

- Additionalfile9.PromptsEmailcommunications.docx 